\title{
Fibromialgia - que abordagem terapêutica?
}

Andreia Neves,* Margarida Valente,* Rita Paulino*

\section{RESUMO}

Objectivos: Identificar os tratamentos farmacológicos e não farmacológicos mais recomendados na abordagem da fibromialgia, com base no nível de evidência científica.

Fontes de dados e métodos de revisão: Revisão bibliográfica na Medline, via plataformas PubMed e Medscape, consulta de livros de texto especializados e textos de apoio fornecidos em congresso, entre 2005 e 2008.

Resultados: Foram seleccionados, com base nos critérios de inclusão, duas normas de orientação clínica, dois artigos de revisão sistemática e 3 artigos de revisão baseada na evidência.

O crescente aparecimento de estudos resultou em novas evidências científicas na abordagem terapêutica desta patologia. As guidelines da «American Pain Society» - 2005, privilegiam a confirmação diagnóstica, a educação do doente, a avaliação e tratamento de co-morbilidades. As guidelines da «European League against Rheumatism» - 2007, acrescentam que as co-morbilidades, o tipo e intensidade dos sintomas são importantes na selecção do tratamento inicial. Os americanos preconizam, como primeira linha, a utilização de antidepressivos tricíclicos e da ciclobenzaprina. Contudo, estudos mais recentes, comprovam a maior eficácia dos inibidores selectivos da recaptação da serotonina e norepinefrina, bem como dos alfa-2-delta ligandos - pregabalina, estes nos doentes com predomínio de distúrbios do sono e ansiedade. Na ausência de resposta clínica à monoterapia, a associação destas duas abordagens é uma hipótese. Ambas as guidelines consideram como fármacos de segunda linha os analgésicos e anti-inflamatórios. Quanto à terapêutica não farmacológica, ambas recomendam o exercício físico e a terapia cognitivo comportamental. É consensual que a combinação da terapêutica farmacológica e não farmacológica, numa abordagem multidisciplinar, deverá ser a primeira opção, ainda que de uma forma individualizada.

Conclusão: A fibromialgia implica um elevado consumo de recursos de saúde, devendo ser abordada perspectivando o indivíduo, enquanto unidade bio-psico-social, e encarada como uma realidade, à luz dos estudos recentes.

Novos estudos deverão ser realizados na tentativa de melhor compreender a fisiopatologia e, assim, desenvolver novas e mais adequadas abordagens terapêuticas.

Palavras-chave: Fibromialgia; Dor crónica; Guidelines; American Pain Society; European League Against Rheumatism.

\section{INTRODUÇÃO}

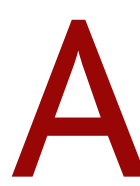

fibromialgia é uma forma não articular e não inflamatória de doença reumática que, de acordo com os critérios de classificação do Colégio Americano de Reumatologia, é caracterizada por dores músculo-esqueléticas generalizadas com pelo menos 3 meses de duração e dor em pelo menos 11 de 18 pontos à palpação digital. Pode associar-se a rigidez prolongada, distúrbios afectivos, alterações do sono e fadiga crónica.

A sua etiopatogénese é desconhecida, havendo estudos que apontam para a interacção de múltiplos processos, num terreno genético propício.

*Internas do $3^{\circ}$ ano de Medicina Geral e Familiar, Centro Saúde de Queluz Unidade Saúde Familiar Mactamã
É mais frequente no sexo feminino, entre os 30 e os 60 anos de idade, sendo a prevalência na população geral de cerca de 2 a $3 \%$.

Dado tratar-se de uma patologia com uma prevalência importante, reconhecida como uma das causas mais comuns de dor crónica e controversa do ponto de vista diagnóstico e terapêutico, cabe ao médico de família saber abordá-la para melhorar a qualidade de vida destes doentes.

Com este trabalho pretendeu-se identificar os tratamentos farmacológicos e não farmacológicos mais recomendados com base no nível de evidência científica.

\section{MÉTODOS}

Foi realizada uma revisão bibliográfica na Medline, via 
plataformas PubMed e Medscape, consulta de livros de texto especializados e textos de apoio fornecidos em congresso, entre 2005 e 2008.

Foram usadas as seguintes palavras-chave: fibromialgia; dor crónica; guidelines; American Pain Society (APS); European League Against Rheumatism (EULAR).

As referências bibliográficas dos artigos foram analisadas para identificar bibliografia adicional. Seleccionaram-se todos os artigos cujo título ou resumo foi considerado relevante, no contexto da revisão em causa. Foram posteriormente obtidos os referidos artigos em texto integral. Incluíram-se nesta revisão duas normas de orientação clínica, dois artigos de revisão sistemática e 3 artigos de revisão baseada na evidência. A selecção foi feita com base na qualidade, abrangência dos conteúdos e na importância relativa dos autores na área considerada.

\section{RESULTADOS}

O tratamento da fibromialgia deve ter como objectivos a diminuição da dor, a melhoria do sono, da condição física e dos restantes sintomas associados e a promoção da reinserção laboral e nas actividades de lazer. O alcance destes objectivos apenas será possível com uma abordagem multidisciplinar envolvendo médicos de família, reumatologistas, fisiatras, fisioterapeutas, psicólogos/psiquiatras, entre outros.

O rápido aumento de estudos sobre a fibromialgia nos últimos anos originou novas evidências científicas na abordagem terapêutica desta patologia, o que permitiu a elaboração de normas de orientação clínica.

Em 2005, foram publicadas normas pela APS, que privilegiam em primeiro lugar a confirmação diagnóstica (painel de peritos) e posteriormente a informação (nível de evidência A) e educação do doente (nível de evidência B), a abordagem multidisciplinar e tratamento combinado da doença (nível de evidência A). ${ }^{1}$

Mais recentemente, em 2007, a EULAR publicou também novas normas de orientação clínica que, por sua vez, defendem uma abordagem multidisciplinar e individualizada do doente fibromiálgico (nível de evidência D), dando importância à avaliação da dor, função e contexto psicossocial (nível de evidência D). ${ }^{2}$

Em relação à terapêutica não-farmacológica, a APS preconiza a realização de terapia cognitivo-comportamental (nível de evidência A), exercício aeróbio mode- rado a intenso (nível de evidência $\mathrm{A}$ ) e fortalecimento muscular (nível de evidência B). A balneoterapia (nível de evidência A), a hipnose clínica, o biofeedback, a manipulação quiroprática, a massagem terapêutica (nível de evidência B) e a acupunctura (nível de evidência C) são também preconizadas por esta instituição na abordagem não-farmacológica da fibromialgia. Sempre que a monoterapia não seja suficiente para o controlo dos sintomas, deve optar-se pela associação de duas ou mais destas técnicas (nível de evidência A). ${ }^{1}$ Por sua vez, a EULAR recomenda a hidroterapia em piscina de água aquecida com ou sem cinesioterapia como um método eficaz (nível de evidência B). O exercício aeróbio, o treino da força muscular, o relaxamento, a fisioterapia e $\mathrm{o}$ apoio psicológico, de acordo com as necessidade individuais, podem ser benéficos (nível de evidência C). Apenas nalguns casos a terapia cognitivo-comportamental pode ser útil (nível de evidência $C$ ). ${ }^{2}$

Segundo a APS, o tratamento farmacológico de primeira linha inclui a utilização de antidepressivos tricíclicos (amitriptilina 10-30 mg ao deitar) e de ciclobenzaprina (nível de evidência A). Podem também ser utilizados os inibidores selectivos da recaptação da serotonina (SSRI's), isolados ou em combinação com os primeiros (nível de evidência B). Se o quadro clínico for dominado por distúrbios do sono, deve dar-se prioridade ao uso de hipnóticos e ansiolíticos (nível de evidência A). Para o alívio da dor, pode usar-se o tramadol (nível de evidência B) na dose de 50-100 mg (bid), que deve ser aumentada progressivamente e nunca suspensa de forma súbita. Este pode ser usado isoladamente ou em associação com o paracetamol (nível de evidência B). Não devem ser utilizados anti-inflamatórios não- esteróides como tratamento de primeira linha para o alívio da dor (nível de evidência A). Os opióides deverão ser reservados para os doentes que não respondam à terapêutica analgésica já referida (painel de peritos). O uso de corticóides não está recomendado (nível de evidência A) a não ser que exista inflamação articular concomitante. ${ }^{1}$

Nas normas de orientação clínica da EULAR está preconizada a utilização de antidepressivos, devido à sua acção na diminuição da dor e na melhoria da função, sendo opções: amitriptilina (tricíclico), fluoxetina (SSRI), duloxetina e milnacipran (inibidores da recaptação da serotonina e norepinefrina - SNRI's), moclo- 


\begin{tabular}{|c|c|c|}
\hline & $\begin{array}{c}\text { APS }^{*}-2005 \\
\text { (nível de recomendação) }\end{array}$ & $\begin{array}{c}\text { EULAR }^{* *}-2007 \\
\text { (nível de recomendação) }\end{array}$ \\
\hline Medidas gerais & $\begin{array}{l}\text { - Iniciar tratamento após confirmação diagnóstica } \\
\text { (painel de peritos) } \\
\text { - Educar doente (B), abordagem multidisciplinar e } \\
\text { tratamento combinado (A) }\end{array}$ & $\begin{array}{l}\text { - Avaliação da dor, função e contexto } \\
\text { psicossocial (D) } \\
\text { - Abordagem multidisciplinar e } \\
\text { individualizada (D) }\end{array}$ \\
\hline $\begin{array}{l}\text { Tratamento Não } \\
\text { Farmacológico }\end{array}$ & $\begin{array}{l}\text { - Informar doente (A) } \\
\text { - Terapia cognitivo-comportamental (A) } \\
\text { - Exercício aeróbio moderado a intenso (A), } \\
\text { fortalecimento muscular (B) } \\
\text { - Hipnose clínica e biofeedback (B), acupunctura (C), } \\
\text { manipulação quiroprática, massagem terapêutica (B) } \\
\text { e balneoterapia (A) } \\
\text { - Ausência resposta monoterapia } \rightarrow \text { terapêutica } \\
\text { combinada (A) }\end{array}$ & $\begin{array}{l}\text { - Hidroterapia (B) } \\
\text { - Exercício aeróbio e treino de força muscular } \\
\rightarrow \text { programa individualizado (C) } \\
\text { - Terapia cognitivo-comportamental em } \\
\text { casos específicos (D) } \\
\text { - Técnicas de relaxamento, fisioterapia, apoio } \\
\text { psicológico } \rightarrow \text { individualizado (C) }\end{array}$ \\
\hline $\begin{array}{l}\text { Tratamento } \\
\text { Farmacológico }\end{array}$ & $\begin{array}{l}\text { - } 1^{\text {a }} \text { linha: antidepressivos tricíclicos e } \\
\text { ciclobenzaprina (A) } \\
\text { - Inibidores da recaptação da serotonina, isolados ou } \\
\text { em combinação com antidepressivos tricíclicos (B) } \\
\text { - Não usar anti-inflamatórios não esteróides como } \\
1^{\text {a }} \text { linha (A) } \\
\text { - Tramadol (B); pode associar-se com o paracetamol (B) } \\
\text { - Uso de opióides ¥ última linha (consenso de peritos) } \\
\text { - Uso de hipnóticos e ansiolíticos } \rightarrow \text { distúrbios } \\
\text { - do sono (A) }\end{array}$ & $\begin{array}{l}\text { - Antidepressivos: amitriptilina, fluoxetina, } \\
\text { duloxetina, milnacipran, moclobemida, } \\
\text { pirlindole (A) } \\
\text { - Outros fármacos que diminuem a dor: } \\
\text { tropisetron, pramipexole, pregabalina (A) } \\
\text { - Tramadol no alívio dor (A); outras opções: } \\
\text { outros opioides fracos e paracetamol (D); } \\
\text { opioides fortes e corticoides não } \\
\text { recomendados (D) }\end{array}$ \\
\hline
\end{tabular}

*APS - American Pain Society; **EULAR - European League against Rheumatism.

bemida e pirlindole (inibidores da monoaminoxidase) - nível de evidência A. Pelo seu efeito analgésico, estão ainda recomendados o tropisetron (antagonista dos receptores do 5HT3), pramipexole (agonista dos receptores da dopamina) e pregabalina (alfa2delta ligando) (nível de evidência A). O tramadol é o principal analgésico preconizado (nível de evidência A). Como outras opções para o alívio da dor indicam outros opióides fracos e o paracetamol (nível de evidência D), não estando recomendados opióides fortes e corticóides (nível de evidência D). ${ }^{2}$

Em 2006 foi publicado um trabalho de revisão de Lesley M. Arnold et al ${ }^{3}$ que sugere a modificação de algumas recomendações da APS, nomeadamente a reconsideração dos antidepressivos tricíclicos como primeira linha de tratamento, uma vez que são fármacos pouco tolerados e que em baixas doses não são eficazes no tratamento da depressão e ansiedade, duas comorbilidades comuns nestes doentes. $\mathrm{Na}$ abordagem da dor moderada a intensa, preconiza, como primeira linha, a utilização de SNRI's - milnacipram (até uma dose máxima de $200 \mathrm{mg} /$ dia) e duloxetina (60 mg 1-2 vezes/dia), uma vez que melhoram a dor, a função, o bem-estar geral e a qualidade de vida destes doentes. Nos indivíduos com distúrbios do sono a autora defende a utilização de pregabalina na dose de 300 a 450 $\mathrm{mg} /$ dia ou gabapentina na dose de 1200 a $2440 \mathrm{mg} /$ dia. A primeira teve aprovação em Junho de 2007 pela Food and Drug Administration para o tratamento da fibromialgia, por diminuir a dor e melhorar a fadiga e o sono. Já a segunda, apesar de ser estrutural e farmacologicamente idêntica à pregabalina, a sua eficácia não foi for- 
malmente demonstrada no tratamento da fibromialgia e tem mais efeitos adversos. No caso de resposta parcial aos SNRI's ou aos alfa2delta ligandos em monoterapia, dever-se-á utilizar a associação de ambos. Em caso de falência desta terapêutica combinada, pode optar-se pelos SSRI's, antidepressivos tricíclicos em monoterapia ou associados, e ainda pela combinação de SSRI's com os alfa2delta ligandos.

Mais recentemente, em Junho de 2008, foi publicado um outro trabalho de revisão, por Srinivas Rao e colegas, do qual se destaca a indicação para o uso de miorelaxantes com eficácia comprovada na melhoria do sono, fadiga e dor - ciclobenzaprina (10-30 mg/dia) e tizanidina (4-24 mg/dia). ${ }^{4}$

\section{CONCLUSÕES}

A fibromialgia implica um elevado consumo de recursos de saúde, devendo ser abordada perspectivando o indivíduo, enquanto unidade bio-psico-social, e encarada como uma realidade, à luz dos estudos recentes. O médico de família deve estar alerta para o diagnóstico e tratamento desta patologia, dada a sua prevalência e impacto na qualidade de vida dos doentes.

A maior probabilidade de sucesso terapêutico advém de uma abordagem multidisciplinar e simultaneamente de um tratamento individualizado, que inclui a utilização de fármacos que actuam essencialmente ao nível do sistema nervoso central.

Novos estudos deverão ser realizados na tentativa de melhor compreender a fisiopatologia desta doença e, assim, desenvolver novas e mais adequadas abordagens terapêuticas.

\section{AGRADECIMENTOS}

Às orientadoras de formação das autoras:

Dra. Fátima Arnaud,* Dra. Rosa Bretes, ${ }^{*}$ Dra. Ana Frazão*

*Assistente Graduada de Medicina Geral e Familiar na Unidade de Saúde

Familiar de Mactamã - Centro de Saúde de Queluz

\section{REFERÊNCIAS BIBLIOGRÁFICAS}

1. American Pain Society. Guideline for management of fibromyalgia syndrome pain in adults and children. Disponível em: http://www.guideline.gov/summary/summary.aspx?ss=15\&doc_id $=7298 \& \mathrm{mpr}=$ 4342 [acedido em 19/05/2008].

2. Barclay L. EULAR issues guidelines for the treatment of fibromyalgia syndrome. Sep 2007. Disponível em: http://www.medscape.com/viewarticle/56316 [acedido em 19/05/2008].

3. Arnold LM. Biology and therapy of fibromyalgia: new therapies in fibromyalgia. Arthritis Res Therapy 2006; 8 (4): 212.

4. Rao SG, Gendreau JF, Kranzler JD. Understanding the fibromyalgia syndrome. Psichofarmacol Bull 2007; 40 (4): 24-67.

5. Burkeham J, Harris ED Jr. Fibromyalgia: a chronic pain syndrome. In: Ruddy S, Harris ED Jr, Sledge CB, editors. Kelley's Texbook of Rheumatology. Philadelphia:W.B. Saunders Company; 2006. p. 522-36.

6. Saraiva F. Fibromialgia. XIV Congresso Português de Reumatologia; 2008 Abr 2-5; Vilamoura - Algarve, Portugal.

7. Jesus H. Os diagnósticos diferenciais da fibromialgia. XIV Congresso Português de Reumatologia; 2008 Abr 2-5; Vilamoura- Algarve, Portugal.

As autoras declararam não possuir conflitos de interesses

\section{ENDEREÇO PARA CORRESPONDÊNCIA}

Andreia Neves

Rua José Régio Lote $1 / 2$, Bloco $A$

$1^{\circ}$ esq - 2700-504 Brandoa

E-mail: andreiasn@hotmail.com

Recebido em 03/06/2009

Aceite para publicação em 21/11/2009 


\section{ABSTRACT}

\section{FIBROMYALGIA - WHICH THERAPEUTIC APPROACH?}

Objectives: To identify the most recommended non-pharmacological and pharmacological treatments for fibromyalgia based on the level of scientific evidence.

Data Source and Review Methods: Literature review in Medline, through PubMed and Medscape, specialized textbook and support literature from congresses consult; between 2005 and 2008.

Results: Based on inclusion criteria, selection was made of two guidelines, two systematic review articles and three evidencebased review articles.

The growing number of studies originates new scientific evidence concerning the therapeutic approach of this disease. The American Pain Society guidelines (2005) highlight diagnostic confirmation, patient education and comorbidities evaluation and treatment. The European League against Rheumatism guidelines (2007) also considers the importance of all comorbidities and symptoms characteristics and intensity when deciding the initial treatment. The Americans defend, as first line therapy, tricyclic antidepressants and cyclobenzaprine. However, more recent studies confirm the higher efficacy of selective serotonin and norepinephrine reuptake inhibitors as well as the alfa2delta ligands - pregabalin, the latter being preferred when the clinical picture is dominated by sleep disturbances and anxiety. The absence of a clinical response to monotherapy should bring to consideration the association of these two approaches. Both guidelines consider analgesics and anti-inflammatory drugs as a second line pharmacological treatment. Concerning non-pharmacological treatment, both guidelines recommend physical exercise and cognitive-behavioural therapy.

The combination of pharmacological and non-pharmacological treatment as a part of a multidisciplinary approach should always be the first option, having in mind each individual case.

Conclusion: Fibromyalgia implies a high cost in health resources. Therefore it should be approached considering the individual as a bio-psycho-social unit, and be faced as a reality, regarding recent studies.

More investigation should be made to better understand the physiopathology of this disease. Only a better comprehension of fibromyalgia will allow the development of newer and more adapted therapeutic options.

Keywords: Fibromyalgia; Chronic pain; Guidelines; American Pain Society, European League against Rheumatism. 